\title{
Measurements of Fluctuating Pressures on a Circular Cylinder in Subsonic Crossflow
}

\author{
J. R. Ackerman* \\ University of Leicester, Leicester LE1 7RH, United Kingdom \\ J. P. Gostelow亡 \\ National Research Council of Canada, Ottawa K1A OR6, Canada \\ A. Rona \\ University of Leicester, Leicester LE1 7RH, United Kingdom \\ and \\ W. E. Carscallen $\stackrel{\S}{-}$ \\ National Research Council of Canada, Ottawa K1A OR6, Canada \\ DOI: $\underline{10.2514 / 1.40954}$
}

\begin{abstract}
A circular cylinder is tested in crossflow over the subsonic speed range. Time-resolved pressure distributions give information on surface pressure fluctuations; the corresponding drag and base drag coefficients are provided. Measured base pressure fluctuations at low Mach numbers are in agreement with the findings of other researchers. Flow changes at higher subsonic velocities and into the transonic range are described. Illustrations are drawn from the observations of other researchers, enabling physical explanations to be given to assist toward a more general modeling of the problem. At Mach numbers above 0.6 the changing strength of the vortices reduces the base drag coefficient up to a Mach number of 0.9 , at which the onset of sonic flow increases the drag. Strouhal number variation is compared with the measurements of other authors. The paper concentrates on providing reliable measurements of vortex shedding and base pressure over the subsonic speed range rather than attempting to provide universal correlations.
\end{abstract}

\section{Nomenclature}

$C_{\mathrm{BD}}=$ base drag coefficient

$C_{D}=$ drag coefficient

$C_{p}=$ static pressure coefficient

$f \quad=$ frequency, $\mathrm{Hz}$

$M a=$ Mach number

$P \quad=$ total pressure, $\mathrm{Pa}$

$p \quad=\quad$ static pressure, $\mathrm{Pa}$

Re $=$ Reynolds number

$S t=$ Strouhal number

$s=$ root mean square value

$\theta=$ cylinder azimuth, deg

\section{Subscripts}

$p=$ phase-averaged pressure coefficient data

$s=$ cylinder surface conditions

sep $=$ time-averaged location of boundary-layer separation

$t=$ time average

$01=$ freestream stagnation condition

$02=$ wake stagnation condition

$180=$ cylinder trailing edge

\section{Introduction}

$\mathbf{T}$ HIS paper reports on an experimental investigation of fluctuating pressures and base pressure variation on a circular cylinder in crossflow over the subsonic speed range. The initial context and motivation emerged from work on a family of gas turbine nozzle vanes with a blunt trailing edge [1]. Denton and $\mathrm{Xu}$ [2] established that for such blades this area of reduced static pressure around the trailing edge was a major contributor to total pressure loss. Turbine blades with a thick trailing edge, such as those investigated by Carscallen et al. [3], had a high loss penalty associated with the trailing edge. This loss penalty was greater than would have been expected from a simple backward-facing step and had been quantified by Deych et al. [4] and Craig and Cox [5].

The high losses remained unexplained until high-speed schlieren photography was applied to cascades [6]. In subsonic flow past a blunt trailing-edged turbine blade, or past the more straightforward geometry of a circular cylinder, periodic vortex shedding is almost always present up to a Mach number of about 1.2. Vortex shedding was found to be present in the wakes of blades with thick trailing edges, often associated with pressure waves propagating upstream along the blade surfaces, when the local freestream flow was subsonic [6]. Hoerner [7] previously correlated base drag for airfoils and backward-facing steps; he found that the base pressure of an airfoil was consistently lower than that of an equivalent backwardfacing step. He attributed this discrepancy to the existence of a von Kármán vortex street in the wake of the airfoil. For turbine blades, Cicatelli and Sieverding []ㅡ conducted an investigation into the effect of vortex shedding on the surface pressure distribution around the cylindrical trailing edge of a linear cascade turbine stator blade using a surface-mounted pressure transducer. They found that the pressure in this region fluctuated by as much as $8 \%$ of the downstream dynamic head near separation and by $4.8 \%$ in the base region. This seemed to indicate that the instantaneous base pressure could be significantly different from the time-averaged value, giving poor computational results if steady-state methods, such as conventional Reynolds-averaged Navier-Stokes, were used. It has been concluded [9] that, for bluff body flows, "calculation methods which neglect base pressure effects are incapable of accurately calculating the flow patterns or the total pressure loss."
Seattle, WA, 23-26 June 2008; received 12 September 2008; revision received 7 April 2009; accepted for publication 26 April 2009. Copyright ( 2009 by the American Institute of Aeronautics and Astronautics, Inc. All rights reserved. Copies of this paper may be made for personal or internal use, on condition that the copier pay the $\$ 10.00$ per-copy fee to the Copyright Clearance Center, Inc., 222 Rosewood Drive, Danvers, MA 01923; include the code 0001-1452/09 and \$10.00 in correspondence with the CCC.

*Department of Engineering.

$\dagger$ Principal Research Officer; also Emeritus Professor, University of Leicester, Leicester, LE1 7RH, United Kingdom. Senior Member AIAA.

'Lecturer, Department of Engineering. Member AIAA.

${ }^{\S}$ Manager of Research and Technology, GTL, IAR. Member AIAA 
Despite the importance of trailing-edge vortex shedding for turbine blading, this problem is a more general one. It was considered important to reduce the study to the most significant variables and to attempt to address the problem in a relatively generic form. For this reason it was decided to simplify the boundary conditions to an isolated and rigidly mounted circular cylinder in subsonic crossflow.

The base pressure is known to be strongly dependent on the Mach number. At supersonic speeds, the main cause of low base pressure is the strong spatial variations of pressure through shocks and expansions; this tends to be a relatively steady process. At subsonic speeds, shocks only play a role as the velocity reaches critical levels. In general, the unsteady process of vortex shedding is more important. These are, therefore, two quite distinct flow regimes. This investigation focuses exclusively on the subsonic speed range and the attendant flow fluctuations.

The classical von Kármán vortex street is the most usual shedding mode at subsonic speeds and results in predictable configuration and frequency characteristics. These vary with the thickness of the boundary layers at separation and, therefore, with Reynolds number. Unlike in the case of a turbine blade, the time-averaged boundary layers on either side of the circular cylinder are symmetrical and sufficiently thin not to smear the shed vortices.

In the current investigation [10], wake traverses of total pressure and temperature were taken and will be the subject of a further publication. In addition to the wake traverses, surface pressure readings from around the cylinder were also taken in an effort to increase the understanding of the vortex shedding and its relationship with the base pressure of circular cylinders.

Data on the flow past circular cylinders at high subsonic speeds are quite limited, and the purpose of this paper is to extend that rather sparse data set. Systematic modeling is ultimately required, however. This calls for a greater physical understanding of the rather complex phenomenon of vortex shedding and its effect on the surface pressure fluctuations in the periodically separated flows of the base region.

\section{Facility and Instrumentation for Circular Cylinder Testing}

The National Research Council (NRC) $1.5 \mathrm{~m}$ trisonic blowdown wind tunnel in Ottawa was used for this investigation, with a twodimensional flow working section fitted. The test section, shown in Fig. 1, was $0.381 \mathrm{~m}$ wide by $1.524 \mathrm{~m}$ high and $3.581 \mathrm{~m}$ long. The air supply comprised three $143 \mathrm{~m}^{3}$ tanks that could be pressurized up to $2.13 \mathrm{MPa}(21 \mathrm{~atm})$. In this mode, the wind tunnel had a discharge Mach number range of 0.1-0.9 and was run at a constant Reynolds

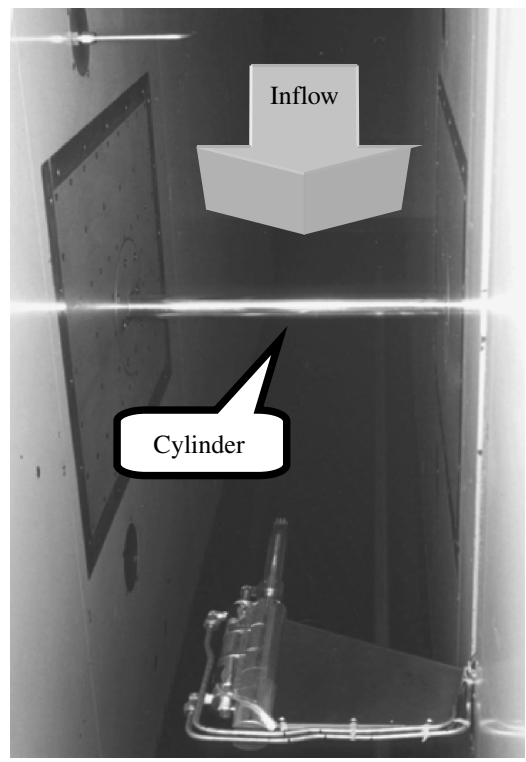

Fig. 1 Cylinder and wake probe mounted in wind tunnel. number of $6.83( \pm 0.03) \times 10^{5}$. The Reynolds number was based on the cylinder diameter and freestream inflow velocity. The operating Reynolds number range and run time were dependent on the Mach number and set by adjusting the total and static pressure of the test section. Blowdown operation resulted in the freestream total temperature dropping over the course of each run as the air in the supply tanks expanded. The model was mounted $2.388 \mathrm{~m}$ downstream of the end of the contraction on reinforced mounts in a threecomponent balance. Because of the method of reinforcement, the balance could not be used to measure the forces acting on the cylinder but it did provide a convenient means of rotationally indexing the cylinder. The balance could be rotated through a range of $\pm 55 \mathrm{deg}$ at a rate of $15 \mathrm{deg} / \mathrm{s}$. Tunnel-wall boundary-layer control was applied using ejector-augmented sidewall suction through $0.602 \times 0.452 \mathrm{~m}^{2}$ porous plates.

Two series of tests were conducted, in 2000 and 2002, respectively, and the results of the surface pressure measurements were designated accordingly. All cylinder surfaces were polished to a hydraulically smooth finish. In the 2000 series of tests, the model was an aluminum circular cylinder with a diameter of $37.26 \mathrm{~mm}$ and a span of $381 \mathrm{~mm}$. A single 0.062-in.-diam Kulite XCQ-062$25 \mathrm{D}$ ultraminiature pressure transducer was mounted so that the B screen, which covered the pressure sensitive diaphragm, was flush with the cylinder surface at midspan. For the 2002 series, similar-sized steel cylinders were used and four 0.093-in.-diam Entran transducers were mounted at equally spaced distances around the circumference.

In the 2000 series of tests, the freestream turbulence level increased from $0.24 \%$ at Mach 0.4 to $0.26 \%$ at 0.6 and $0.34 \%$ at 0.8 . Subsequent modifications have reduced these levels marginally for the 2002 series.

A tube-mounted Kulite XCQ-062-25D ultraminiature pressure transducer was carried in a wake probe used for traversing downstream of the cylinder. The pressure transducer was mounted in a conventional pitot configuration for total pressure measurement such that its face pointed directly into the flow. The sensitivity to flow direction was that of a conventional pitot tube, giving a $1 \%$ error for a $10 \mathrm{deg}$ misalignment. The probe sting was mounted for wake traverse using a triangulated support. The sting and support were designed so that the probe tip would be 6 cylinder diameters or $223.6 \mathrm{~mm}$ downstream of the model's leeward-most point, as shown in Fig. 1.

Cross sections of the cylinders used for the 2000 and 2002 test series are shown schematically in Fig. 2. Surface pressure measurements were taken in 5 deg increments from 10 deg below the leading edge to $10 \mathrm{deg}$ below the trailing edge. The forward stagnation point was the zero location, and the azimuthal orientation was such that the angle increased in a clockwise direction. The cylinder could be rotationally indexed, thus minimizing the number of surface pressure transducers required. Data were taken for $2 \mathrm{~s}$ at each position. Each surface pressure traverse was taken over two blowdowns. In the first of these, the cylinder was traversed from $10 \mathrm{deg}$ below the leading edge to around $100 \mathrm{deg}$ above it. The second traverse covered the 80190 deg locations.

Alongside each surface pressure reading, a 2 s record was taken from the wake probe pressure transducer that acted as a phase reference. Phase reference locations were chosen for pressure measurements with strong signals that were dominated by the vortexshedding fundamental frequency. The signals were low-pass filtered at $40 \mathrm{kHz}$, for antialiasing, before being recorded at a sample rate of $100 \mathrm{kHz}$ directly onto the PC through National Instruments data acquisition cards.

\section{Results and Discussion}

\section{A. Surface Pressure Distribution}

Figures $\underline{3}$ and 4 show time-averaged surface pressure distributions for the circular cylinders, taken from the 2000 and 2002 series of tests, respectively. The surface pressures are presented in the form of the pressure coefficient: 


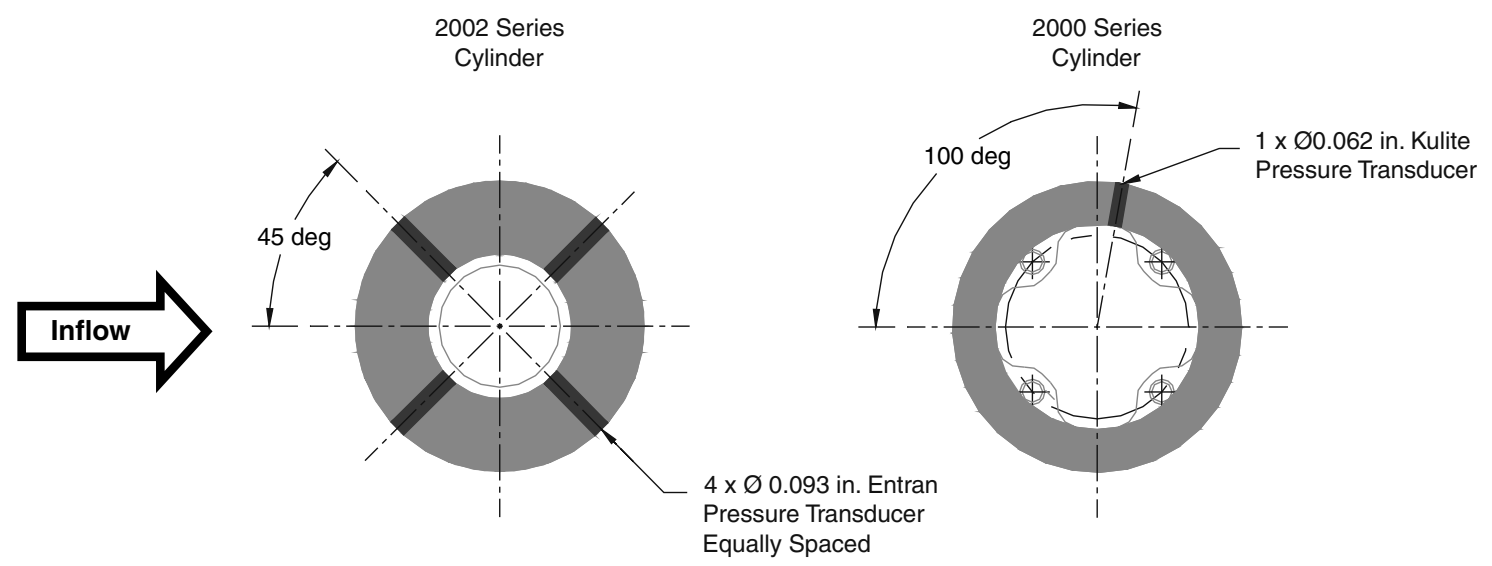

Fig. 2 Location of pressure transducers within cylinder models.

$$
C p_{t}=\frac{p_{s, t}-p_{1}}{P_{01}-p_{1}}
$$

Superimposed on the mean values, $\left\langle C_{P}\right\rangle_{t}$, are the maximum and minimum levels of the raw pressure coefficient, $C_{P}$, and the rms of the raw and phase-averaged fluctuations in $C_{P}$. The rms of the pressure coefficient for both the raw and phase-averaged data is
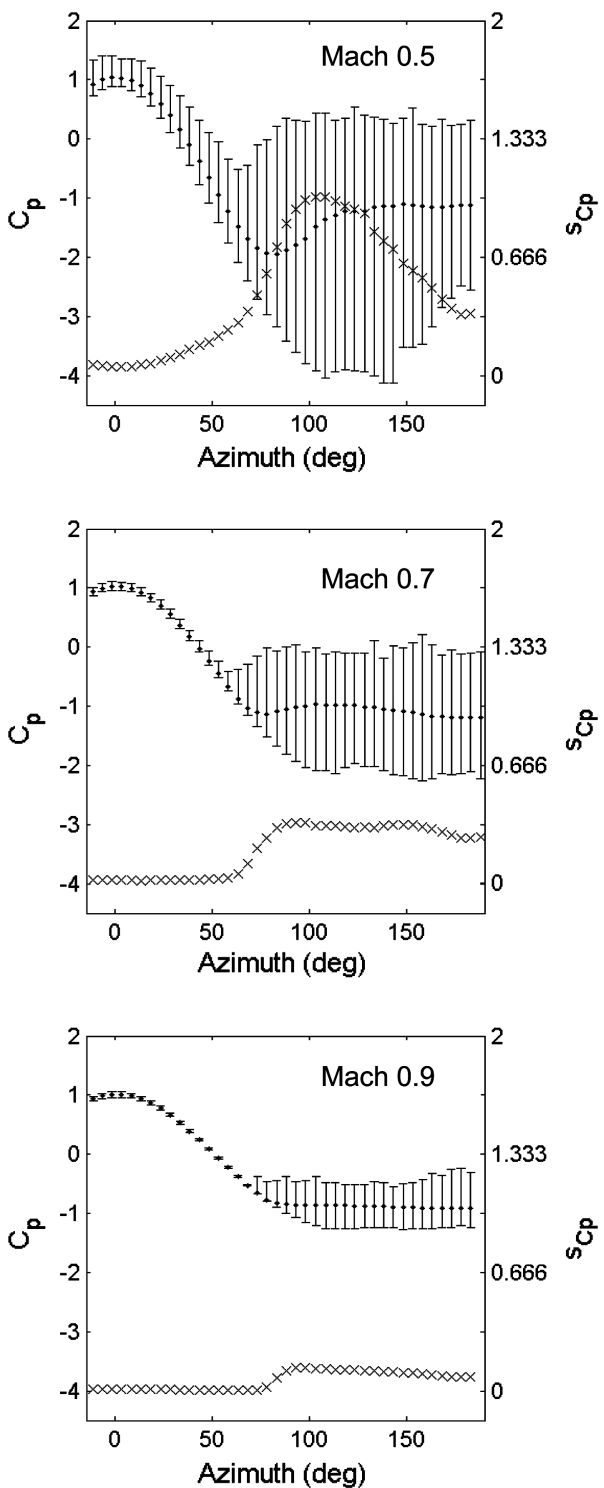

Fig. 3 Pressure distributions from the 2000 series data. where $X$ is the quantity being examined, and $N$ is the number of time samples.

Figures 3 and 4 show that the pressure distributions at a Mach number of $\overline{0} .5$ are broadly similar in the 2000 and 2002 test series.
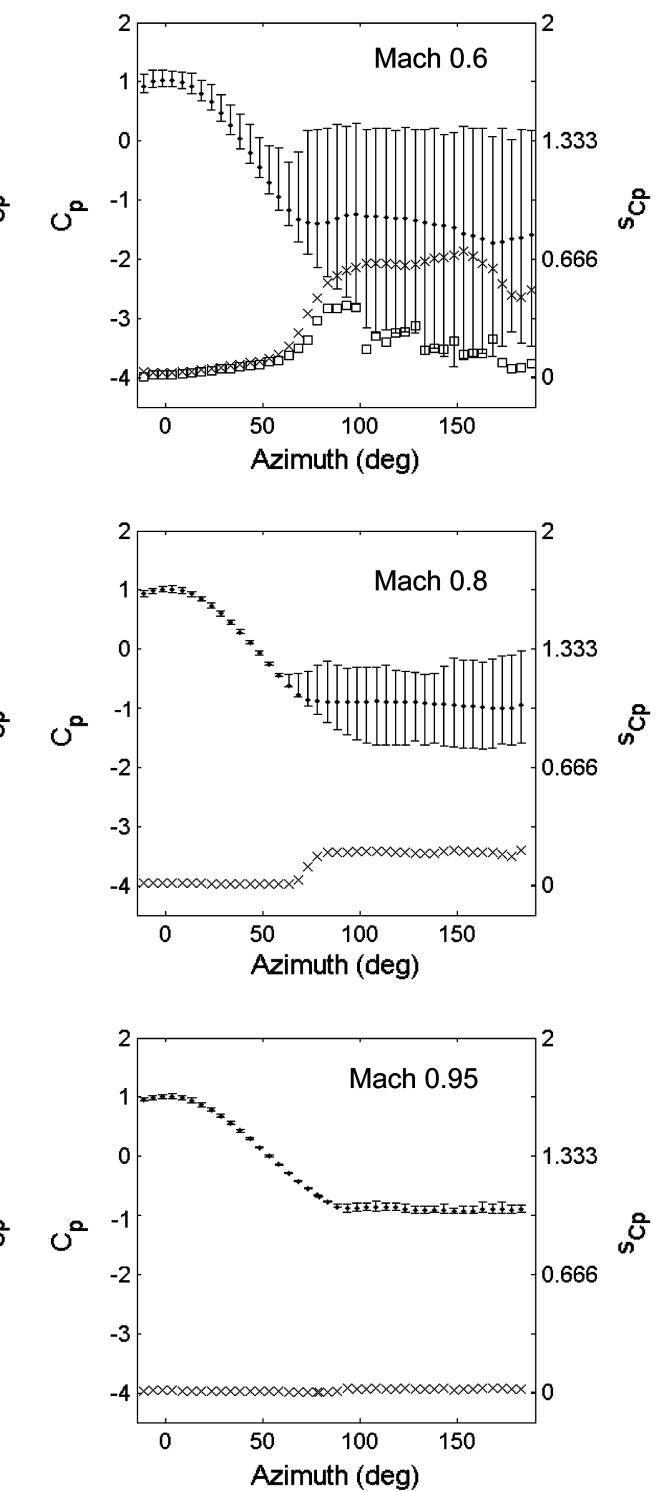

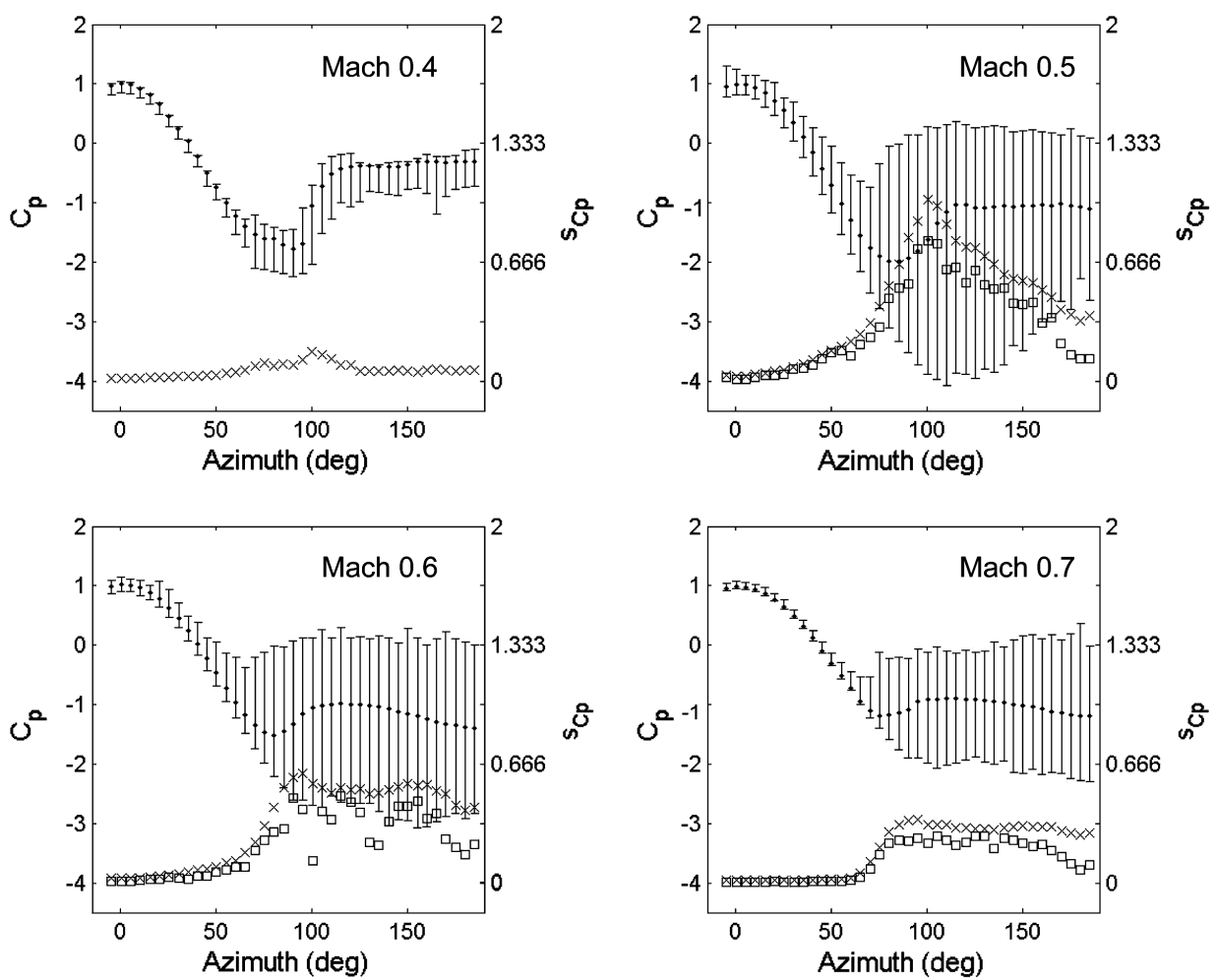

Fig. 4 Pressure distributions from the 2002 series data.

The same observation applies to the Mach 0.7 results. However, at Mach 0.6 there are considerable differences between the results of the two series. These are most significant over the azimuthal angle range from 90 to $110 \mathrm{deg}$. The most probable cause of such a difference is a change in the way the circular cylinder was instrumented in the two experiments. In the 2000 series, one surface pressure transducer was used, located as sketched in Fig. 2, whereas in the 2002 series four pressure transducers were used, evenly distributed around the cylinder circumference at midspan. As the cylinder is rotated to measure the surface pressure at different azimuthal angles, the positions of the pressure transducers change the effective geometry of the test model. Specifically, whereas in the 2000 series the flow sees a smooth cylindrical surface upstream of the measurement location, in the 2002 series the flow is perturbed by the presence of the upstream pressure transducer. This is likely to have affected the boundary-layer tripping, as will be discussed in Sec. III.D. The tripping of the cylinder surface boundary layer affects its separation and the pressure recovery on the leeward surface over the Mach number range from 0.5 to 0.7 . Figures $\underline{3}$ and $\underline{4}$ indicate that the flow is particularly sensitive at Mach 0.6.

\section{B. Drag and Base Drag Coefficients}

The change in base pressure distribution at Mach 0.6 can best be quantified by finding the coefficients of form drag, $C_{D}$, and base drag, $C_{\mathrm{BD}}$, defined in Eqs. (3) and (4), for the various profiles and plotting them against the Mach number, as in Fig. $\underline{5}$.

$$
\begin{gathered}
C_{D}=\int_{\theta=0}^{\theta=180} C p \cos \theta \cdot \mathrm{d} \theta \\
C_{\mathrm{BD}}=\int_{\theta=\mathrm{sep}}^{\theta=180} C p \cos \theta \cdot \mathrm{d} \theta
\end{gathered}
$$

This method of calculating profile drag includes contributions from form drag only and neglects skin friction and drag created by the loss of total pressure across any local shock wave that may be present around the cylinder surface. At a Reynolds number of $6.83 \times 10^{5}$, the contribution to the profile drag from skin friction drag is very small
[11]. Given that the flow is subsonic and transonic, the contribution to the drag by total pressure loss through shocks will be small, although it will increase, along with the discrepancy caused by neglecting it, as the Mach number approaches unity.

\section{Compressible Flow Regimes}

Before considering the differences between the two sets of data, an explanation of the changes in $C_{D}$ over the Mach number range will give insight into the reason for the differences. From Fig. 5 it can be seen that both $C_{D}$ and $C_{\mathrm{BD}}$ start low and then rise to a maximum at Mach 0.6, before falling off at Mach 0.7 and then rising again slowly at the higher Mach numbers. These variations over the Mach number range are compatible with the compressible flow regimes described by Zdravkovich [11], namely, shockless, intermittent shock wave, permanent shock wave, and wake shock wave. Figure 6 is adapted from the work of Dyment et al. [12] (Mach 0.45 and 0.64) and

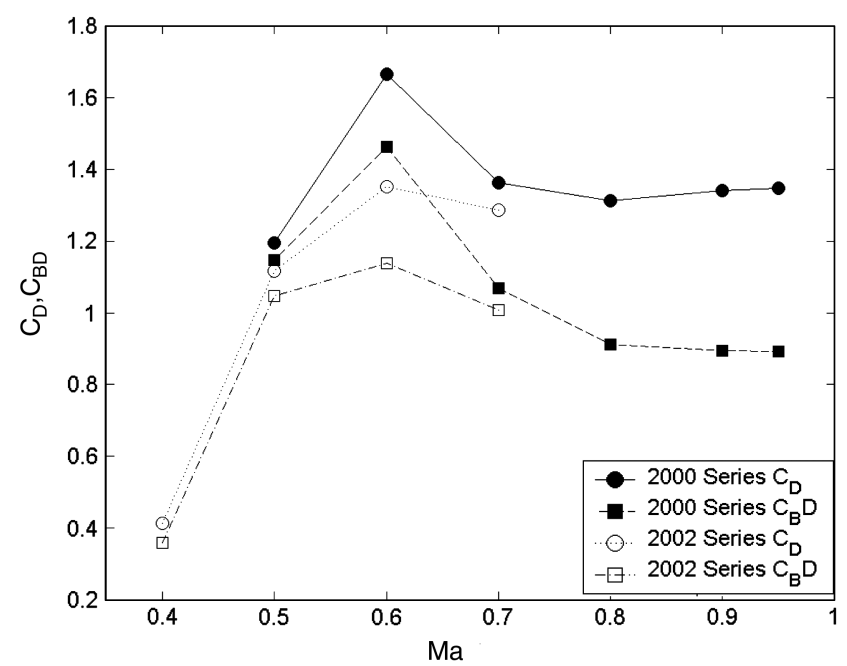

Fig. 5 Drag and base drag coefficients over the Mach number range of $0.4 \leq M a \leq 0.95$. 


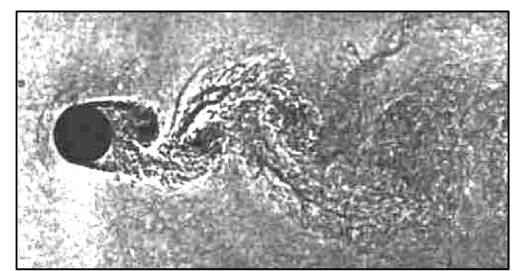

a) Shockless [12] - Mach 0.45

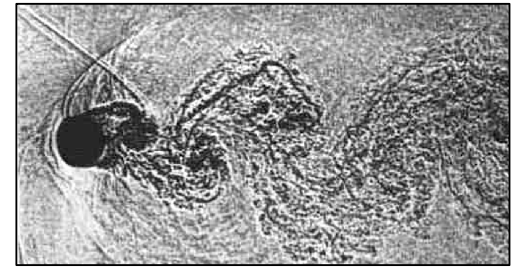

b) Intermittent shock [12] - Mach 0.64

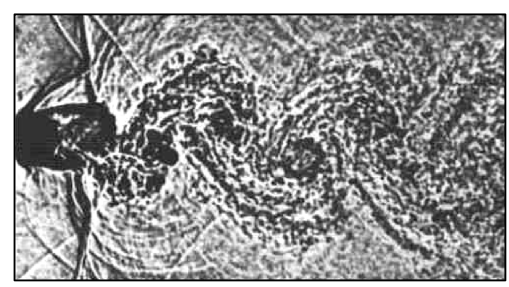

c) Permanent shock [13] - Mach 0.80

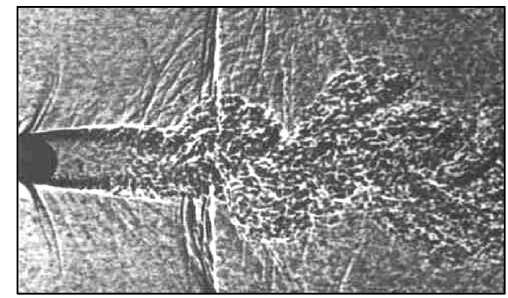

d) Wake shock [13] - Mach 0.90
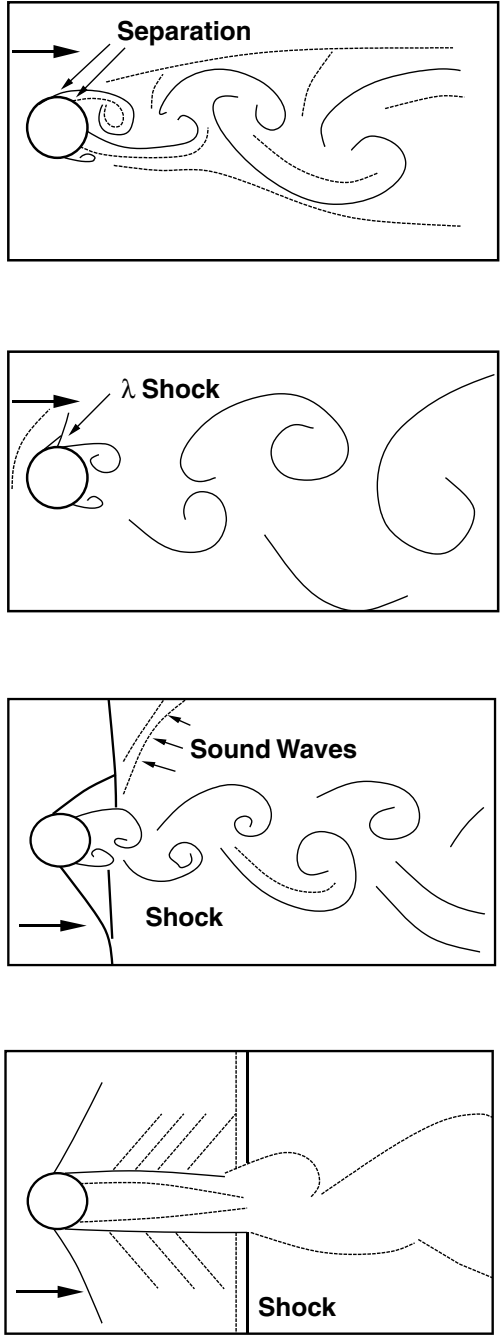

Fig. 6 Illustration of evolving shock/shear-layer interactions for Mach numbers between 0.45 and 0.90 . Adapted from the observations of Dyment et al. [12] and Dyment and Gryson [13].

Dyment and Gryson [13] (Mach 0.80 and 0.90) and illustrates the different regimes.

At Mach 0.4, the flow is in the shockless regime and, therefore, is strongly Reynolds-number dependent; it is this that determines $C_{D}$. In this case, given the Reynolds number used in the test, the flow falls into the transitional boundary layer 3 (TrBL3) subsonic regime [11]. This explains the lack of vortex shedding and low $C_{D}$ observed at this Mach number: "Further increase in $\operatorname{Re}$ [into the TrBL3 regime] brings transition to the primary laminar separation line in an irregular manner. This leads to the disruption and fragmentation of separation bubbles along the span of the cylinder. The irregularly fragmented separation line prevents eddy [vortex] separation..." [11]. The lack of vortex shedding permits the high level of pressure recovery, seen at Mach 0.4 in Fig. 4, which results in the low $C_{D}$.

Between Mach 0.4 and 0.5 the flow becomes critical; local regions of flow about the cylinder become momentarily supersonic and the flow enters the intermittent shock wave regime. The oscillating flowfield present in this regime, which results in the flow being supersonic only on one side of the cylinder at a time, leads to increased pressure fluctuations compared with the previous Mach number. This can be clearly seen in the change in the rms level and the maximum and minimum pressure levels seen in Fig. 4, at freestream Mach numbers of 0.4 and 0.5 , respectively. The surface pressure coefficient at which the local isentropic Mach number is unity for a Mach 0.4 inflow is lower than the minimum surface pressure coefficient reported in Fig. 4. Hence, the Mach 0.4 inflow of the 2002 series is subcritical. Similar considerations indicate that the flow was momentarily supersonic for an inflow Mach number of 0.5 .
As the freestream Mach number increases beyond the critical limit, the region behind the cylinder in which the vortices are formed shortens. The increase in $C_{D}$ leading up to Mach 0.6 is a result of the commencement of vortex shedding, the shortening of the vortexshedding region with increasing Mach number, and the formation of the local shock waves as the intermittent shock wave regime strengthens. Beyond around Mach 0.65 the flow enters the permanent shock wave regime, shown in Fig. $6 \mathrm{c}$. The permanent shock wave regime features the movement of the location of formation and the shedding of the vortices downstream of the cylinder surface. The vortices and the shocks are a coupled system. The shocks are inevitable but they couple with a free shear layer, oscillating longitudinally with the shocks, to give vortex roll up and shedding. This lengthens the formation region, increases the pressure recovery, and gives a slightly earlier separation. This, in turn, leads to the reduction in $C_{D}$ at Mach 0.7.

At just below Mach 0.8, the flow typically enters the wake shock wave regime. This occurs at a slightly higher Mach number in Dyment and Gryson [13]. Once the flow enters the wake shock wave regime, at just below Mach 0.8 , the vortex formation region becomes elongated; a normal shock grows at the point of vortex roll up, and the drag on the cylinder increases. This increase can be seen in Fig. 5 . As the flow moves further into the wake shock regime at Mach $0.9 \overline{5}, C_{D}$ again increases slightly and no vortex shedding is detected on the surface of the cylinder.

Figure $\underline{5}$ also highlights the discrepancy in $C_{D}$ between the two sets of results at Mach 0.6. Below and above Mach 0.6, the differences in $C_{D}$ between the two sets of results are 0.08 at Mach 0.5 
and 0.07 at Mach 0.7. These differences are small, approximately 7 and $5 \%$ of the overall $C_{D}$ at Mach 0.5 and 0.7 , respectively, and can be attributed to experimental error and the slight change in Reynolds number, as the Mach number has not yet increased sufficiently to remove all Reynolds number effects. However, at Mach 0.6 the difference is 0.31 ; this is quite considerable when one recalls that at Mach $0.4 C_{D}$ is 0.41 , suggesting that there is a difference between the boundary-layer flows in the 2000 and 2002 series results.

\section{Pressure Distributions}

To aid the investigation into the $C_{D}$ discrepancy, the 2000 series pressure profiles are shown in Fig. 7 with the 2002 series profiles superimposed for Mach numbers of $0.5,0.6$, and 0.7 .

\section{Base Pressure Data}

In Fig. 7, at Mach 0.5, the 2002 series time-averaged surface pressure distributions, denoted by open circles, follow closely the 2000 series distributions from the upstream stagnation point $(\theta=0 \mathrm{deg})$ to an azimuthal angle of about $100 \mathrm{deg}$. In the range of $100 \mathrm{deg} \leq \theta \leq 130 \mathrm{deg}$, there is a greater static pressure recovery in the 2002 series data than in the 2000 series data. The difference indicates the presence of an early laminar separation in the 2000 series tests, whereas the separation in the 2002 series tests is delayed by about $10 \mathrm{deg}$ of azimuth. This effect is more pronounced at Mach 0.6 (Fig. 7), at which the flow separates at $\theta \approx 90 \mathrm{deg}$, causing a reduction in base pressure that affects the entire leeward face
(90 deg $\leq \theta \leq 180 \mathrm{deg}$ ). This separation is accompanied by a larger excursion between the minimum and maximum pressure coefficients, indicated by the dashed lines. This is indicative of very strong vortex shedding in the 2000 series tests. The Mach 0.7 pressure distribution (Fig. 7) also shows a greater pressure recovery at $\theta \approx 95 \mathrm{deg}$ for the 2002 series tests. This shows that for all three Mach numbers the boundary layer at separation is mostly laminar whereas the cylinder boundary layer during the 2002 series tests is either turbulent or undergoing transition at separation. This would lead to an irregularly fragmented separation line in the 2002 series, a feature of the TrBL3 regime highlighted in Sec. III.C, as compared with a more uniform separation and greater spanwise coherence in vortex shedding in the 2000 series. This produces the greater surface pressure fluctuation amplitude recorded in the 2000 series test that is indicative of stronger shedding.

\section{Boundary-Layer Tripping}

The tripping of the boundary layer into a turbulent or transitional state may have happened in one of two ways. The first possibility is that a change in the running conditions of the facility itself between the two sets is the cause of the change. However, the freestream Reynolds numbers for the 2000 and 2002 series tests are virtually identical, at $6.86 \times 10^{5}$ and $6.80 \times 10^{5}$, respectively. Measurements taken during the latter set of tests are thought to be those of a cylinder with a tripped boundary layer. Tests of the freestream pressure fluctuations conducted on the facility indicate that the fitting of a new control valve port ring shortly after the 2000 series tests actually
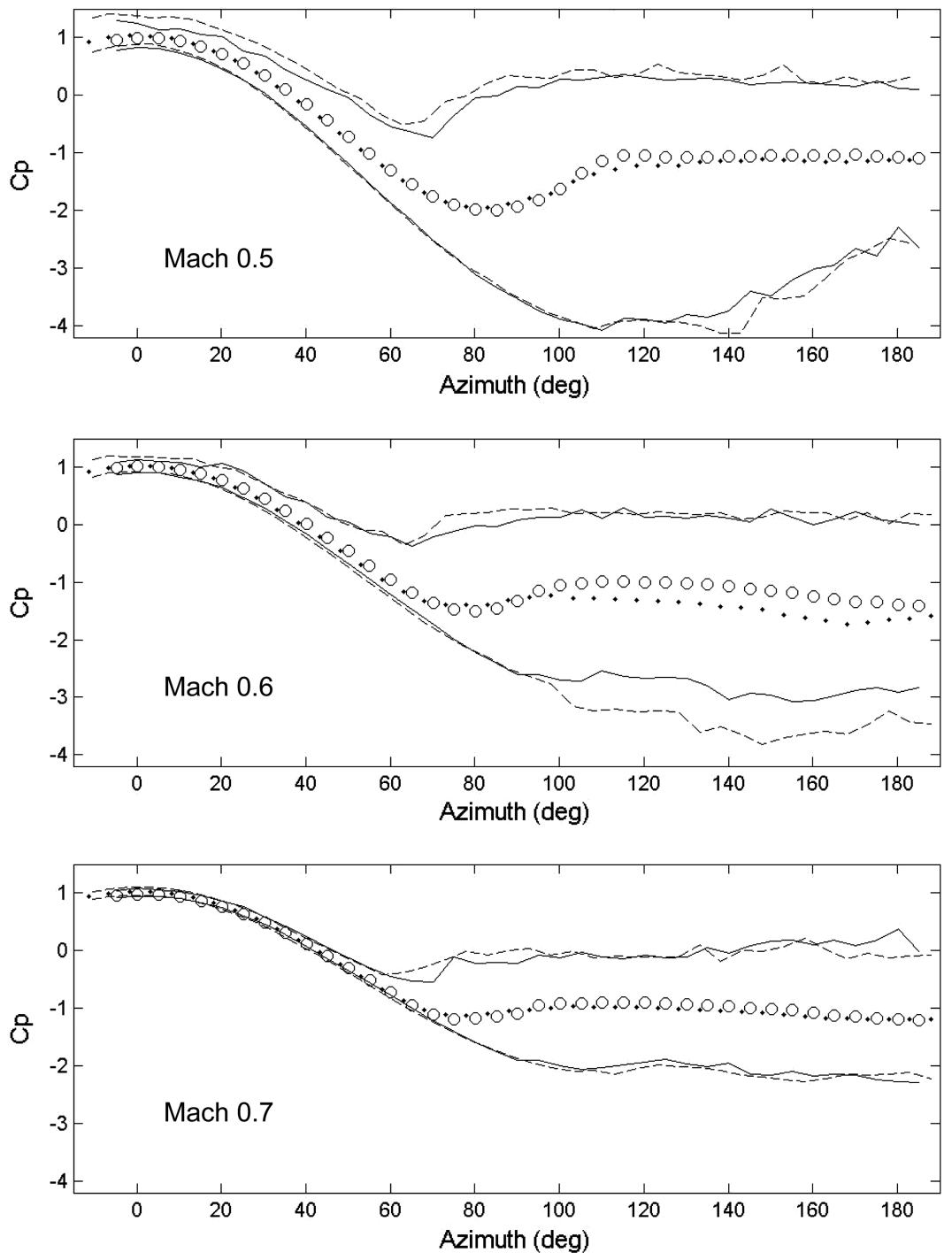

Fig. 7 Pressure distributions for the 2000 and 2002 series tests. 
reduced the freestream turbulence by a small margin. This means that, if the only changes between the tests are those to freestream conditions, the boundary layer should have been more laminar during the 2002 series tests than in the 2000 series tests. Clearly, this is not the cause of the change in separation position and pressure recovery between the two sets of data.

The second possibility is that the presence of four pressure transducers positioned around the midspan of the cylinder in the 2002 series of tests, shown in Fig. 2, may have tripped the boundary layer. The 2000 test series only has a single transducer of smaller diameter. The presence of the pressure transducers on the surface of the cylinders presents flat spots, the transducer diaphragm making the surface curvature discontinuous. Although this is minimized by fitting each transducer so that the face is flush with the upstream and downstream edges of the mounting hole, discontinuities are inevitably formed, with the larger transducer creating the larger discontinuities. Because of the larger diaphragm diameter, the presence of the transducers creates discontinuities in the surface curvature that are larger for the Entran transducers of the 2002 series.

\section{Shock/Shear-Layer Interactions}

By themselves, the discontinuities may or may not cause the boundary layer to become transitional or turbulent. However, when one considers that the critical Mach number for a circular cylinder is just above 0.4, then at the higher Mach numbers of the 2002 series these discontinuities may well cause the creation of local shock waves that cause the boundary layer to trip. This is illustrated schematically in Fig. $\underline{8}$. During the 2000 series of tests, there was only one transducer on the cylinder surface and the discontinuity caused by it was small. This means that any alteration to the boundary layer as a result of shock waves stemming from this small discontinuity occurred after the flow had passed over the transducer. Because any effect this has on the flow is not easily communicated upstream due to the flow being sonic, the readings taken are effectively those of a laminar boundary layer with shock-induced laminar separation.

This was not the case for the 2002 series of tests. The windwardfacing transducers on the cylinder used for the 2002 series tests ensure that the flow seen by the leeward transducers would always be tripped, even with the four pressure transducers placed at an azimuthal position other than the symmetric $45 \mathrm{deg}$ orientation shown in Fig. 2. Thus, the leeward readings are all of a tripped boundary layer with shock-induced turbulent separation. Given that the cylinder wake is mostly determined by the location of flow separation, the flow asymmetry caused by the rotation of the four pressure transducers in the 2002 series tests should be less of an influence on the aerodynamic loads compared with the location of flow separation. Therefore, as long as the boundary layer is tripped upstream of its separation point, the main flow wake should be essentially insensitive to the rotation of the four pressure transducers.

It would seem, then, that the most likely cause of the differences in $C_{D}$ and $C_{\mathrm{BD}}$ between the series of tests is the presence of discontinuities in the surface curvature due to the extra transducers. Although this means that the exact flow conditions for the two sets of results differ, it also means that data exist for vortex shedding from a circular cylinder with both a purely laminar boundary layer and a tripped turbulent or transitional boundary layer. This should be borne in mind during the subsequent discussion of the results.

Despite the differences between the drag coefficients and base drag coefficients taken from the 2000 and 2002 series, both sets

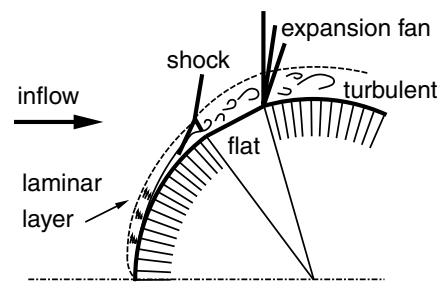

Fig. 8 Schematic representation of the effects of the pressure transducer on the boundary layer. display the same trend. As the vortex shedding becomes established, at Mach 0.5 and 0.6 , there is a significant rise in $C_{D}$ and $C_{\mathrm{BD}}$. Above Mach 0.6, the coefficients fall off at Mach 0.7 and then, in the case of $C_{D}$, rise slowly; in the case of $C_{\mathrm{BD}}$, they fall slowly. The large difference between the coefficients at Mach 0.6 and Mach 0.7 may be attributed to the change in flow regime from intermittent shock wave to permanent shock wave at around Mach 0.65. Given that the coefficients do not change much after Mach 0.7, it can be deduced that the change in the coefficients between Mach 0.6 and 0.7 is due to the flow around the cylinder containing supersonic regions throughout the vortex-shedding cycle. The slight changes after Mach 0.7 are due to delayed separation and greater pressure recovery.

\section{Surface Pressure Fluctuations}

Another point of difference between the two sets of data is the rms levels of the phase-averaged and raw $C_{P}$ at Mach 0.6. The 2000 series results have a notable drop in the level of the rms between the raw and phase-averaged data, which does not occur in the 2002 series data for Mach 0.6 shown in Figs. 3 and 4. The cause of this is the use of wake pressure readings as a phase reference during the 2000 series results. Although the wake readings do provide a reasonable phase reference, there is a greater level of jitter in the wake pressure reading than in those readings taken on the cylinder surface. This has the effect of slightly smearing the phase-averaged data, resulting in a lower rms of the fluctuations; some of the fluctuation feeds into the variance of the phase average in the data processing. This means that, although the 2000 series phase-averaged $C_{P}$ are valid data, they are not of such high quality as the 2002 data. Specifically, in the 2000 series data, some of the physical rms fluctuation due to the flow unsteadiness feeds instead into the variance of the phase locked average in the data processing.

Another flow feature that shows up clearly in Fig. 7 is the drop in the level of pressure fluctuation on the windward side, forward of around the $60 \mathrm{deg}$ azimuth, as the Mach number increases. Inspection of Figs. 3 and 4 shows that this is generally true from Mach 0.5 upward. It is caused by the strengthening of the intermittent and, eventually, permanent supersonic regions on the surface of the cylinder along with the attendant surface shock waves up to the wake shock waves regime. The presence of supersonic regions prevents pressure fluctuations from being communicated upstream during the half period that it is not supersonic; this results in sizable surface pressure perturbations. Thus, in the intermittent shock wave regime during the first half of the shedding cycle, the pressure fluctuations can move forward over one half of the surface and, during the second, they can move forward over the other half. The ability to prevent the fluctuations from moving forward is dependent on the size of the supersonic regions and, therefore, increases with the freestream Mach number as these regions grow.

\section{Isentropic Mach Number Distributions}

The cessation of pressure fluctuations over the windward side of the cylinder at Mach 0.7 indicates that localized areas of supersonic flow are, or are nearly, permanent. This is evidenced in Fig. 9; at Mach 0.6, the time-averaged isentropic Mach number reaches a maximum just above unity and remains there. This indicates that the intermittent shock waves are indeed present for only half of a vortexshedding cycle, allowing the movement upstream of pressure fluctuations during the half of the vortex-shedding cycle that is not supersonic, resulting in sizable surface pressure fluctuations. At Mach 0.6, the maximum isentropic Mach number about the leeward surface appears to reach a very high value at some points. Low values of surface static pressure are observed, but the very high apparent isentropic Mach numbers are an artifact of the data reduction procedures.

At Mach 0.7, the time-averaged isentropic Mach number accelerates to well above unity, indicating that the flow on both sides of the cylinder remains supersonic for a significant proportion of the time. While the flow on both sides of the cylinder is sonic, there can be no, or in practice very little, communication of pressure fluctuations upstream, thus reducing the maximum and minimum 

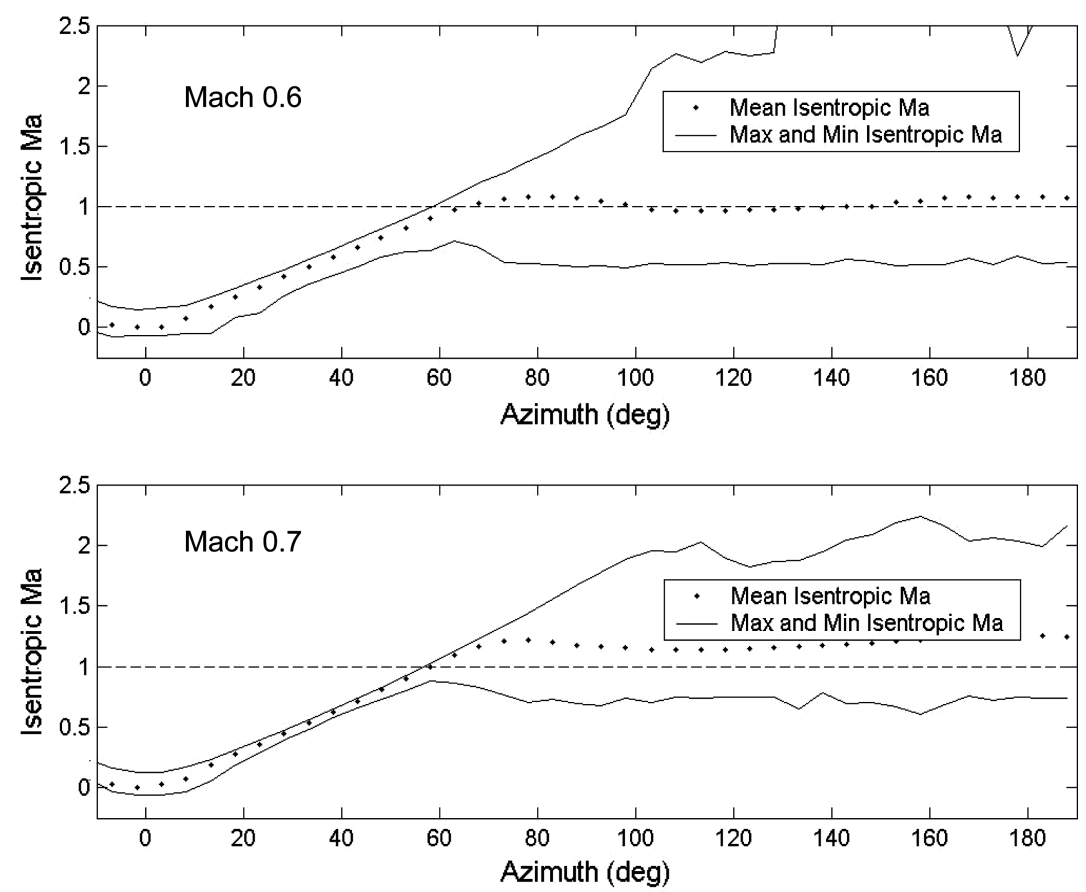

Fig. 9 Isentropic Mach number distribution for 2000 series results.

levels of isentropic Mach number on the windward face. This is shown in Fig. 9 by a reduction in the width of the region delimited by the maximum and minimum isentropic Mach numbers, in the range of $0 \mathrm{deg} \leq \theta \leq 60 \mathrm{deg}$, as the Mach number increases from 0.6 to 0.7 .

\section{E. Vortex Formation and Shedding \\ 1. Shedding Frequency}

The changes in flow features and vortex-shedding mechanisms can be seen from the changes in vortex-shedding frequency with freestream velocity and Mach number, Fig. 10a. From Gerrard [14], the shedding frequency may be viewed as the result of a balance between the size of the formation region and the thickness and state of the shear layers feeding into it. Figure 10a shows that the rate of increase in the fundamental vortex-shedding frequency between Mach 0.6 and 0.8 remains linear. Above Mach 0.8, the rate of increase in vortex-shedding frequency with freestream velocity increases and, below Mach 0.6, initially decreases and then increases down to Mach 0.4. This seems to indicate that either the amount of fluid entrained into each vortex remains the same throughout the Mach 0.6-0.8 range or the rate of entrainment and the amount of fluid entrained changes in such a way as to create a linear increase in shedding frequency. Below Mach 0.6 and above Mach 0.8, either the amount of fluid entrained per vortex changes nonlinearly, or the rate of entrainment changes nonlinearly, or both. The nonlinear increase in shedding frequency at Mach 0.5 is further explored by considering the possibility of flow/acoustic coupling in the wind-tunnel test section. The tunnel acoustic modes closest to the vortex-shedding frequency are the second transversal mode at $892 \mathrm{~Hz}$ and the composite of the first transversal mode and the sixth flow-normal mode at $804 \mathrm{~Hz}$. These acoustic modes are rather remote from the vortex-shedding frequency at Mach 0.5 , so that flow/acoustic coupling is unlikely to have occurred at Mach 0.5 .

The apparently linear increase in shedding frequency between Mach 0.6 and 0.8 is interesting as, in this Mach number range, the flow regime changes twice, from intermittent shock waves to permanent shock waves and then to wake shock waves, although the final change in regime has only just occurred at Mach 0.8 . The formation of vortices, the subsequent increase, up until just over Mach 0.6, and then reduction in their strength and the increase in frequency at which they are shed is very complex and dependent on a number of variables.

A possible reason for the cessation of the linear increase in shedding frequency above Mach 0.8 can be found in the flow visualization of Dyment and Gryson [13]. From their flow visualization at Mach 0.8 and 0.9 , the vortex formation region can be seen to lengthen by around two-and-a-half times. This suggests that when the permanent shock wave regime first appears there is initially little change in the formation of the vortices.

As the flow moves further into the regime, the differences between it and the intermittent shock wave regime become more apparent as the flow features become stronger. Accordingly it seems reasonable to expect the nonlinear increase in shedding frequency to be related to the extension of the vortex formation region. Above Mach 0.9, the vortex formation becomes yet more complex as the shear layers start to converge, causing the vortex formation region to thin. However, as noted next, at this Mach number it is impossible to determine the Strouhal number from surface pressure measurements and so the changes here are unknown.

\section{Frequency Spectra}

The fundamental shedding frequencies are taken from frequency spectra of the data created using MATLAB's ${ }^{\circledR}$ psd function. A sample of these for each Mach number, taken at an azimuth of around 85 deg, is given in Fig. 11.

It is worth noting that there is very little difference in the vortexshedding frequencies found in the 2000 and 2002 series of tests. For example, at Mach 0.6, at which the biggest differences between the two series are found, the vortex-shedding frequencies are 928 and $944 \mathrm{~Hz}$ for the 2000 and 2002 series results, respectively, a difference of just $16 \mathrm{~Hz}$ or $1.7 \%$. At Mach 0.5 and 0.7 , the differences between the two series are $18 \mathrm{~Hz}$ or $2.1 \%$ and $30 \mathrm{~Hz}$ or $2.7 \%$, respectively. This indicates that, although the difference in boundary-layer state does have some effect on the vortex-shedding frequency, it is very small.

It should also be noted that at the Reynolds number used in these tests there should be no vortex shedding at all when the flow is fully subsonic, as it falls into the TrBL3 regime [11]. Indeed, from a quick inspection of the frequency spectra for Mach 0.4 , it appears that this is the case. However, a closer inspection of the data reveals that there is a broad increase in the power spectrum magnitude in the region of 


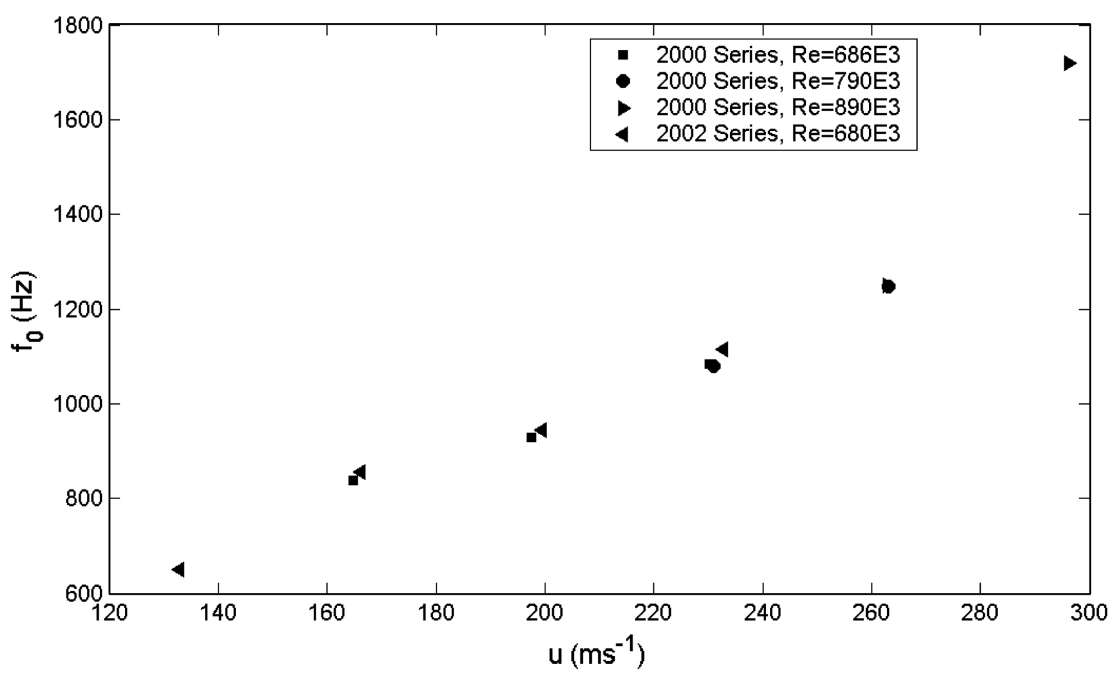

a)

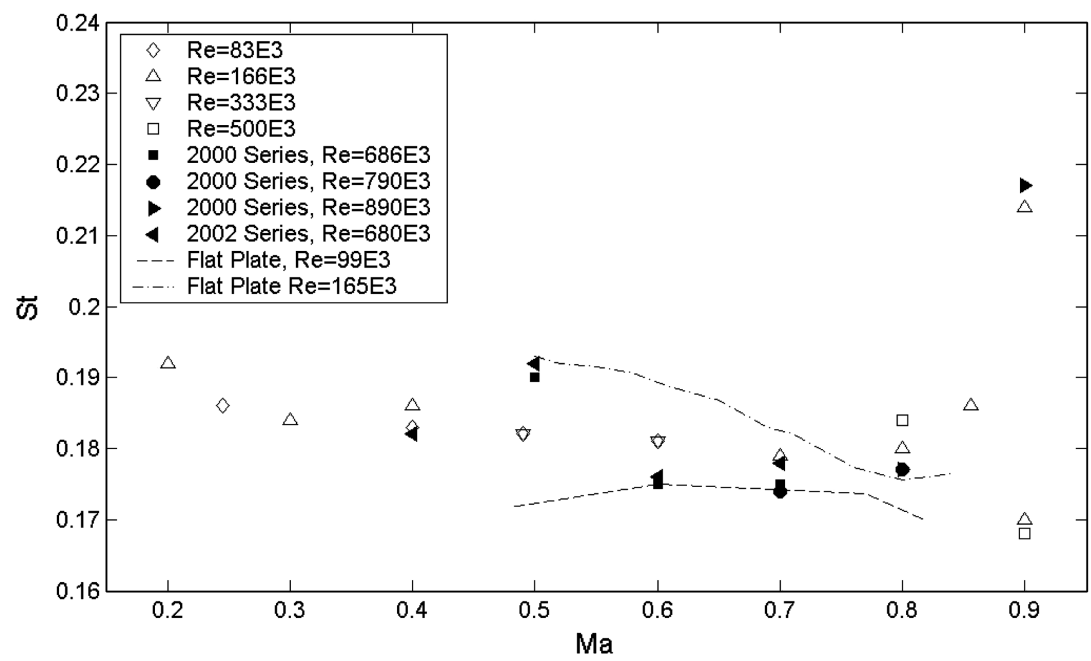

b)

Fig. 10 Vortex-shedding frequency across the experimental range: a) fundamental shedding frequency as a function of freestream velocity, and b) Strouhal number over the Reynolds number range of $0.83 \times 10^{5}<R e>5 \times 10^{5}$. Open symbols: Murthy and Rose [15], flat plate: Heinemann et al. [16].

around $550-670 \mathrm{~Hz}$ at azimuths of around $80-90 \mathrm{deg}$. Also, a closer inspection of the time-resolved data, from around 80 to $90 \mathrm{deg}$, indicates occasional sinusoidal fluctuations with wavelengths that match this broad frequency range. From this it is inferred that there is weak intermittent shedding of vortices at this Mach number. The Reynolds number used is very near the upper boundary of the TrBL2 flow regime, within which vortex shedding does occur. Furthermore, the authors have ample evidence of vortex shedding occurring behind turbine blades under these conditions and much further into the transonic regime [3].

At Mach 0.5, the fundamental vortex-shedding mode is accompanied by two harmonics with about a $20 \mathrm{~dB}$ logarithmic decrement per octave, whereas, at Mach 0.6 and 0.7 there are just the fundamental vortex-shedding mode and one harmonic. The presence of a higher number of harmonics at Mach 0.5 correlates with the nonlinear variation of the Strouhal number over this Mach number range, observed in Fig. 10b, confirming the greater complexity of the fluid dynamic instability at this Mach number.

\section{Strouhal Number}

The frequency data are too sparse to fit a curve that allows for the nonlinear behavior with changing Mach number. However, further insight can be gained into the behavior of the vortex-shedding frequency through inspection of the nondimensionalized vortexshedding frequency, the Strouhal number. When plotted against the
Mach number, as in Fig. 10b, the changes in Strouhal number confirm the indications given by the changes of shedding frequency regarding the shedding mechanisms. This is because, as well as normalizing the data with respect to the cylinder diameter and freestream velocity, the Strouhal number is also the product of the first derivative of shedding frequency with respect to freestream velocity and the cylinder diameter. Thus, any change in the rate of increase of the fundamental vortex-shedding frequency with the freestream velocity becomes clear when the Strouhal number is plotted against the Mach number.

As the Mach number increases from 0.4 to 0.5 the Strouhal number, $S t$, rises from 0.182 to 0.192 as the intermittent shock wave regime is established. $S t$ then falls off significantly from around 0.19 down to about 0.176 between Mach 0.5 and 0.6 as the local supersonic regions become stronger. The Strouhal number then remains fairly constant through the intermittent shock wave and permanent shock wave regimes, confirming the linear increase of shedding frequency in this region, before increasing to 0.217 at Mach 0.9 as the wake shock wave regime strengthens.

These findings are supported by the circular cylinder results of Murthy and Rose [15] and the flat plate work of Heinemann et al. [16] that are plotted alongside the results of the 2000 and 2002 series tests. The results from Murthy and Rose are broadly similar to those from these tests, although they do not show an increase in Strouhal number at Mach 0.5. They do report that their findings at Mach 0.4 show a broadband increase in the frequency spectra, indicating that they had 

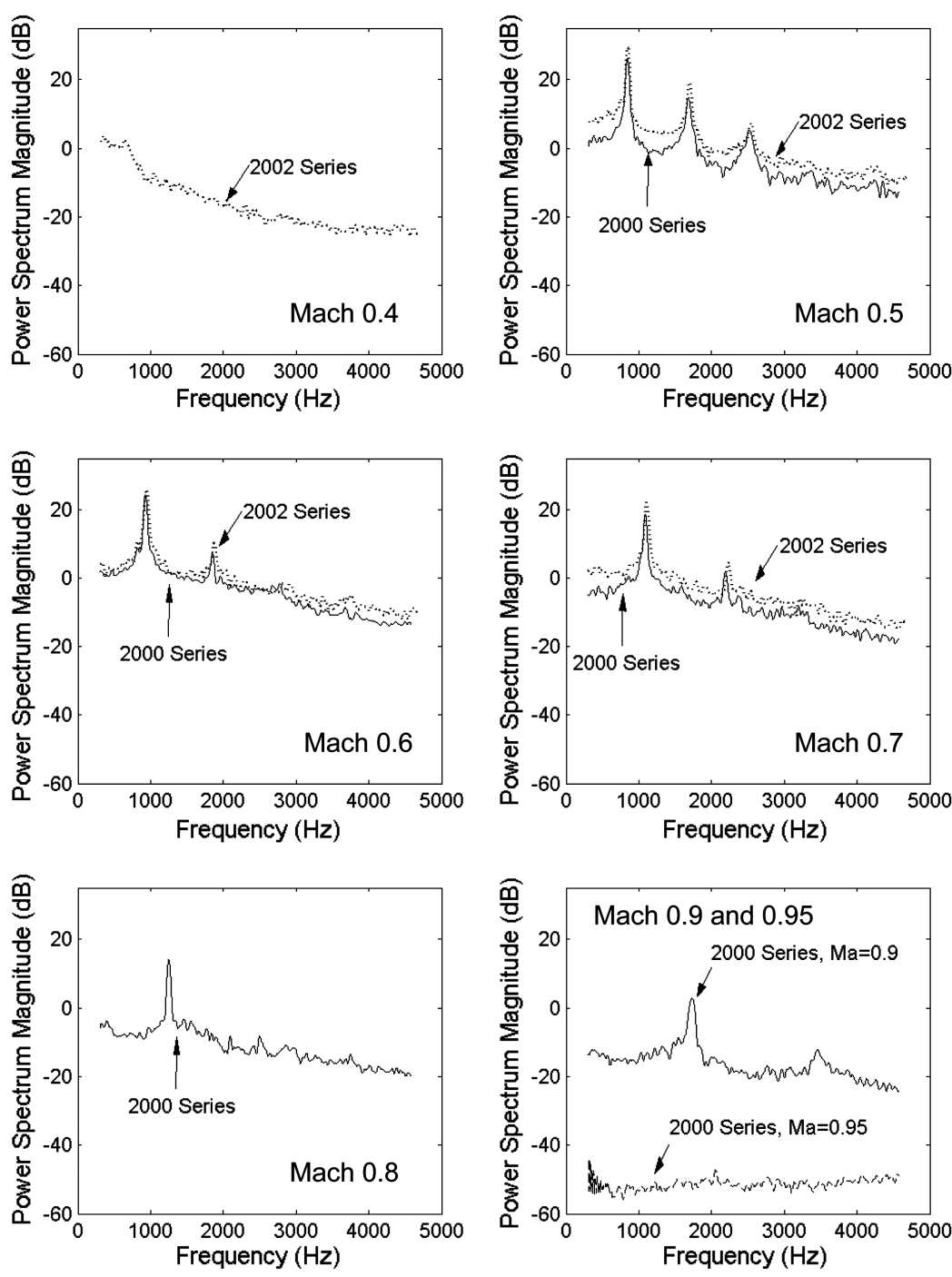

Fig. 11 Frequency spectra of surface pressure results taken at azimuths of $83.21 \mathrm{deg}$ (2000 series) and $84.21 \mathrm{deg}(2002$ series).

similar results to these but interpreted them differently. It is also interesting that at high Mach numbers Murthy and Rose found that the Strouhal number could either rise or fall. This would indicate that the vortex shedding at this Mach number is unstable, confirming the findings of Carscallen et al. [3]. The work of Heinemann et al. [16] is presented by way of comparison between the wake flow of flat plates (and, by extension, airfoils and the blades of turbines and compressors) and that of circular cylinders. Although it is clear from the patterns formed by the different sets of data that the flow regimes for the two types of flow have different limits, this is to be expected given the low critical Mach number of circular cylinders. Regardless of the differences, the similarity between the two sets of data is encouraging.

Another point of interest is that from the data in Fig. 10b there is little difference in the Strouhal number from the data taken at different Reynolds numbers but the same Mach number. This indicates that, once the flow around a circular cylinder has become critical, the Reynolds number ceases to be a major determining factor in the flow. It is also interesting to note that few Strouhal number data are given in the literature for Mach numbers above 0.9. Although Zdravkovich [11] states that no vortex shedding is found above Mach 0.9, the flow visualization [13] that he used at Mach 0.95 indicates that vortex shedding does occur up to that Mach number. The 2000 series surface pressure measurements at Mach 0.95 show no periodic fluctuation. This can be seen from the low rms level and maximum and minimum pressures at Mach 0.95 in Fig. 3. The salient feature is that at Mach 0.95 a strong oblique shock forms at the confluence of the free shear layers from which the vortices are formed and shed
[13]. It seems likely, then, that the supersonic region of flow responsible for this shock prevents pressure fluctuations from moving upstream to the cylinder surface where they could be detected.

\section{F. Summary}

In summary, it may be said that, at Mach numbers above 0.6, the base drag and drag coefficients begin to fall with increasing Mach number until the flow enters the wake shock wave regime. Once there, the drag and the base drag coefficients begin to rise again. In a similar vein, the Strouhal number remains approximately constant from Mach 0.6 up to Mach 0.8, at which the wake shock wave regime begins. It would seem, then, that the mechanism responsible for the formation of large scale vortices downstream of the cylinder remains the same through the intermittent shock wave and permanent shock wave regimes but that, once the steadier near wake of the wake shock wave regime is established, the mechanism changes, leading to increased base drag, despite an increase in pressure recovery.

The change in mechanism can be seen from the change in behavior of the time-resolved data both in the time and frequency domains. Although the magnitude of the pressure coefficient fluctuations around the surface of the cylinder fall in the Mach number range of $0.5-0.7$, the behavior remains the same. The behavior then changes at Mach 0.8. This is marked by a sudden clearing up of the signal, accompanied by a significant drop in the surface pressure fluctuation amplitude. The new behavior, as described in Sec. III.C, indicates a change of mechanism. This is characterized by the disappearance of 
any pressure fluctuations on the windward face of the cylinder, that is, before separation, and an increasingly sharp rise in the frequency content during and after separation, indicating a reduction in the movement of the separation shocks.

The results also show that the state of the boundary layer before separation has little effect on the drag and base drag coefficients except at Mach 0.6. A possible explanation for this is the strength of the intermittent shock wave regime at Mach 0.6. Below this Mach number, the regime is still establishing itself and, above it, it is eradicated by the formation of the permanent shock wave regime. Mach 0.6 is where the difference in the boundary-layer state has the greatest effect on the flow immediately before, during, and just after separation, resulting in the greatest, and indeed only significant, difference between the values of drag measured for the two series of tests. It would seem, then, that the mechanism for the formation of the vortices has a very significant impact on the base drag of the cylinder.

\section{Conclusions}

Time-resolved pressure distributions give information on the location and strength of surface pressure fluctuations on a circular cylinder in crossflow over the subsonic speed range. The corresponding drag and base drag coefficients are presented. Data on Strouhal number variation over the subsonic speed range are also provided and are compared with measurements by other authors.

Measurements have been collected in two measurement campaigns, in which the instrumentation changed from a single surface pressure transducer mounted on the cylinder midspan in the 2000 series to four surface pressure transducers evenly spaced around the circumference at midspan in the 2002 series. As a result, sets of data have inadvertently been produced without and with a boundary-layer trip.

The state of the boundary layer on the cylinder surface influences the vortex shedding. Specifically, the disturbance introduced by the presence of the upstream pressure transducers in the 2002 series is thought to promote an early transition, leading to a turbulent boundary layer that is more tolerant of the adverse pressure gradient on the cylinder leeward surface; this delays boundary-layer separation. As a result, the thickness of the wake and the size of the vortex formation region are reduced and the Strouhal number is affected.

Consequently, the crossflow about a circular cylinder changes significantly at higher subsonic velocities and into the transonic range. The flow regime changes a number of times, resulting in a larger excursion between the minimum and maximum pressure coefficients. This is indicative of very strong vortex shedding in the 2000 series tests. The changing strength of the vortices can also be seen, at Mach numbers above 0.6, to reduce the base pressure deficit and, hence, the drag coefficient up to a Mach number of 0.9 , at which the onset of permanent sonic flow increases the drag acting on the cylinder. At this higher Mach number, a normal shock develops across the wake downstream of the cylinder that becomes the location of the onset of disturbances at Mach 0.9. As vortices form downstream of the normal shock, their pressure perturbation cannot travel upstream through the supersonic flow toward the circular cylinder surface, where the surface pressure fluctuation amplitude is attenuated with respect to that at Mach numbers between 0.5 and 0.8 .

This paper has concentrated on providing some detailed data on pressure fluctuations rather than attempting to produce universal correlations. It was considered more important to provide detailed information and physical explanations of the various configuration changes when possible. This should be of assistance in eventually providing a more general model of vortex shedding on a circular cylinder in crossflow over the subsonic range and its effects on parameters such as base pressure.

\section{Acknowledgments}

A research grant from the Engineering and Physical Sciences Research Council is gratefully acknowledged. The authors are indebted to the NRC for the use of the U-66 wind tunnel and workshop facilities, without which this investigation would have been impossible. Appreciation is expressed to the U-66 team for their excellent support of the work throughout the operation of the facility and to Steve Zan, Ernest Hanff, and Paul Hunt, and others at NRC and Leicester for providing help, support, and expert advice.

\section{References}

[1] Williamson, R. G., and Moustapha, S. H., "Annular Cascade Testing of Turbine Nozzles at High Exit Mach Numbers," Journal of Fluids Engineering, Vol. 108, No. 3, 1986, pp. 313-320.

[2] Denton, J. D., and Xu, L., "The Trailing Edge Loss of Transonic Turbine Blades," Journal of Turbomachinery, Vol. 112, 1990, pp. 277-285. doi:10.1115/1.2927648

[3] Carscallen, W. E., Currie, T. C., Hogg, S. I., and Gostelow, J. P., "Measurement and Computation of Energy Separation in the Vortical Wake Flow of a Turbine Nozzle Cascade," Journal of Turbomachinery, Vol. 121, No. 4, 1999, pp. 703-708. doi:10.1115/1.2836723

[4] Deych, M. E., Fillipov, G. A., and Lazerev, L. Y. A., Atlas of Axial Turbine Cascade Characteristics, Mash Publishing House, Moscow, 1965.

[5] Craig, H. R. M., and Cox, H. J. A., "Performance Estimation of Axial Flow Turbines," Proceedings of the Institution of Mechanical Engineers Part A, Journal of Power Engineering, Vol. 185, 1970-1971, pp. 407-424.

[6] Lawaczeck, O., and Heinemann. H. J., "Von Kármán Streets in the Wakes of Subsonic and Transonic Cascades," CP 177, AGARD, Neuilly-sur-Seine, France, 1976, p. 28-1.

[7] Hoerner, S. F., Fluid Dynamic Drag, published by the author, Vancouver, WA, 1958.

[8] Cicatelli, G., and Sieverding, C. H., "The Effect of Vortex Shedding on the Unsteady Pressure Distribution around the Trailing Edge of a Turbine Blade," American Society of Mechanical Engineers Paper 96GT-359, 1996.

[9] MacMartin, I. P., and Norbury, J. F., "The Aerodynamics of a Turbine Cascade with Supersonic Discharge and Trailing Edge Blowing," American Society of Mechanical Engineers Paper 74-GT-120, 1974.

[10] Ackerman, J. R., "Unsteady Energy Separation and Base Pressure Distributions in Subsonic Crossflow around a Circular Cylinder,’ Ph.D. Thesis, Univ. of Leicester, 2004.

[11] Zdravkovich, M. M., Flow Around Circular Cylinders, Vol. 1: Fundamentals, Oxford Univ. Press, Oxford, 1997.

[12] Dyment, A., Gryson, P., and Ducruet, C., "Unsteady Separation and Reversed Flow in External Fluid Dynamics," Euromech 1397, Marseilles, France, 1980.

[13] Dyment, A., and Gryson, P., "Study of Turbulent Subcritical and Supercritical Flows by High-Speed Visualisation," AGARD Paper 28, 1979.

[14] Gerrard, J. H., "The Mechanism of the Formation Region of Vortices behind Bluff Bodies," Journal of Fluid Mechanics, Vol. 29, Part 2, 1967 , pp. 259-272. doi:10.1017/S0022112067000795

[15] Murthy, V. S., and Rose, W. C., "Form Drag, Skin Friction, and Vortex Shedding Frequencies for Subsonic and Transonic Cross Flows on Circular Cylinder," Proceedings AIAA 10th Fluid and Plasma Dynamics Conf., Albuquerque, AIAA, New York, 1977, pp. 677-687.

[16] Heinemann, H.-J., Lawaczeck, O., and Bütefisch, K. A., "Von Kármán Wakes and Their Frequency Determination in the Wake of Profiles in Sub- and Transonic Regimes," IUTAM Symposium Transsonicum II, Springer, Berlin, 1975. 